\title{
Heat Treatment of Mill-hardened Beryllium Copper for Space Applications
}

\author{
Mitesh Mankani, S S Sharma* \\ Manipal Institute of Technology, Manipal University, India
}

Copyright (C) 2015 by authors, all rights reserved. Authors agree that this article remains permanently open access under the terms of the Creative Commons Attribution License 4.0 International License

\begin{abstract}
Beryllium-Copper (BerylCo) alloys serve as a suitable material for fabrication of satellite antennas due to their high 'strength to weight' ratio and favourable electrical properties. Conventionally, satellites use BerylCo strips that are at least $10 \mathrm{~mm}$ wide. During the designing of nanosatellites certain dimensional constraints prevail that restrict the width of the BerylCo strip to $2 \mathrm{~mm}$. This poses various problems as a thinner Berylco strip, when coiled, loses its ability to retain the original shape upon uncoiling. Thus, the yield strength of the Beryllium-Copper strip must be increased by Heat Treatment. The method of Heat Treatment that is to be employed for the same is Solution Annealing followed by Ageing or Precipitation. However, while carrying out this Heat Treatment process, a problem is faced during the quenching phase because the thin strip experiences rapid heat dissipation into the air. In this paper, an attempt is made to adopt the conventional Heat Treatment method with suitable modifications to overcome the limitations.
\end{abstract}

Keywords Nano-satellites, Beryllium-copper, Heat Treatment, Solution Annealing, Aging

\section{Introduction}

Conventional Satellites are rapidly being replaced by Nano-Satellites today. Due to technical advancements, Nanosatellites are able to carry out the same functions as conventional satellites at a significantly lower cost. The construction of a nanosatellite offer several challenges, one of which is the fabrication of the antenna.

The material generally used for fabrication of satellite antennae is Beryllium-Copper or BerylCo. BerylCo offers high strength at low weight and its electric properties support the transfer of communication signals between the satellite and the ground station on earth making it a favorable choice. [1]

Generally BerylCo strips having a minimum width of $10 \mathrm{~mm}$ are used. These strips of are coiled before launch and they uncoil after detumbling in space. The nanosatellite must be designed to meet the dimensional constraints of its launch vehicle. Thus, in certain cases the width of the strip is required to be as low as $2 \mathrm{~mm}$. The reduction in width causes the strip to lose its ability to regain its original shape when uncoiled. It is possible to overcome this problem by increasing the yield strength of the BerylCo strip, which can be achieved by a heat treatment process of solution annealing followed by Ageing. This process involves a technique called quenching where the heated test specimen is to be cooled in a specific medium before it drops below a critical temperature. However, it has been experimentally observed that once the strip is removed from the furnace, it dissipates heat very rapidly. Thus, its temperature falls below the critical temperature limit before the quenching process can begin. Additionally, it is important for the strip to be uncoiled and held in tension before quenching.

Hence, $\mathrm{Be}-\mathrm{Cu}$ in the binary alloy solution can be age hardened to improve its mechanical properties, especially hardness and strength. The maximum saturation solubility of $\mathrm{Be}$ in $\mathrm{Cu}$ is $16.5 \%$ by weight. Any Copper Beryllium alloy with $\mathrm{Cu}$ in the system less than $10.4 \%$ by weight is generally age hardenable. [2]

Age Hardening is the conventional heat treatment process for non-ferrous metals. It is similar to conventional hardening or tempering treatment of steels [3,4]. Age hardening (precipitation hardening) involves two steps known as solutionizing (solid annealing) and ageing.

Solutionizing comprises of heating the two phase room temperature structure to single phase solid solution region and quenching to room temperature. This gives high temperature single phase structure existing at room temperature as a supersaturated solid solution. The ageing step comprises of holding the specimen at constant temperature well below the solvus temperature but well above room temperature. Holding at constant temperature causes the solute atoms to diffuse out from the saturated solid solution to form new solute rich intermetallic phase. Lower the temperature, better is the hardness $[5,6]$. Once peak hardness is attained in the specimen, it is quenched again to room temperature to arrest the diffusion process causing 
hardness and strength to increase. The toughness obtained depends upon the distribution of the solid rich phases $[7,8]$.

The treatment is also given to a wide variety of Aluminum alloys like 2XXX, 6XXX and 7XXX series. Also, Titanium alloys, Niobium alloys may be subjected to age hardening to enhance the wear resistance and tensile properties. Metal matrix composites of above mentioned alloy matrix may also respond positively for the treatment $[9,10]$.The aim of this paper is to highlight the processes involved in increasing the yield strength of BerylCo strips as well as the problems encountered while doing so. The paper further suggests solutions for the same.

\section{Heat Treatment Method}

It should be noted that due to the presence of alloying elements or impurities in the Beryllium-Copper sustem, the phase diagram may not accurately represent the exact transformation temperatures. The equilibrium diagram should be considered as a guide only. The alloy considered is Berylco Alloy no.14 (C17510). The chemical composition is shown inTable. 1

Table 1. Chemical composition of $\mathrm{Cu}-\mathrm{Be}$ Alloy

\begin{tabular}{|c|c|}
\hline Element & Weight Percentage \\
\hline $\mathrm{Be}$ & $0.2-0.6$ (actual : 0.420$)$ \\
\hline $\mathrm{Cu}$ & 99.5 \\
\hline
\end{tabular}

The process of precipitation hardening is carried out in two stages 'Solution Annealing' and 'Ageing'.

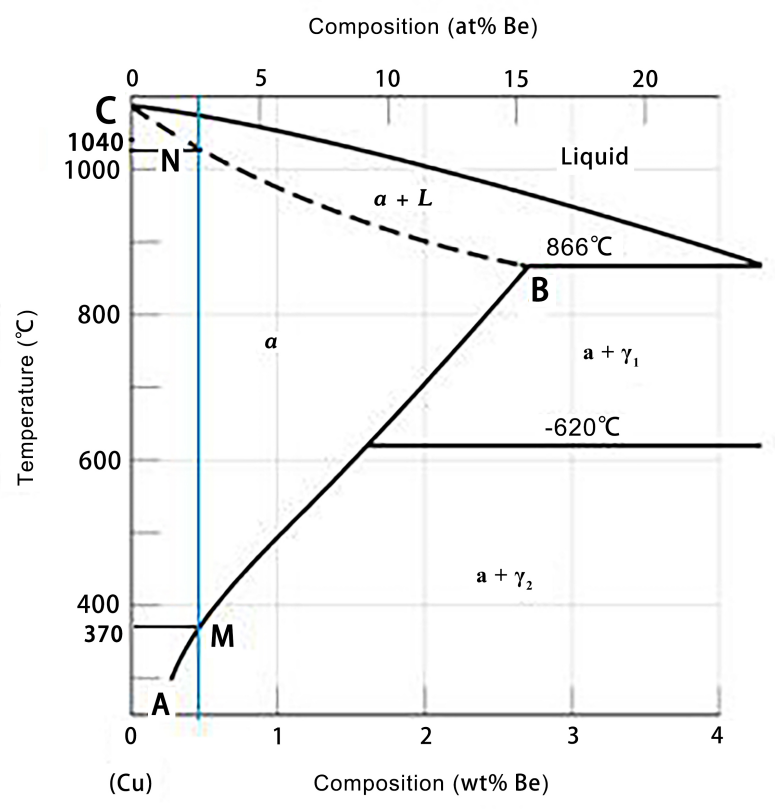

Figure 1. Phase diagram of Beryllium Copper alloy

\subsection{Solution Annealing}

The material is heated to a temperature of approximately $650^{\circ} \mathrm{C}$ for about 15 minutes. The maximum temperature up to which the specimen should be heated at this stage is indicated by the intersection of the curve $\mathrm{BC}$ (shown by the dotted line in Figure1) and the line representing percentage weight composition of Beryllium in the Copper Beryllium solid solutions (Figure 1). At this temperature the beryllium dissolves in the copper matrix to form single phase solid solution. In order to maintain this solid structure at room temperature as super saturated solid solution, the strip is water quenched.

\subsection{Ageing}

Ageing increases the strength of the material. This stage is carried out at $350^{\circ} \mathrm{C}$, followed by quenching. The minimum temperature to which the specimen should be heated in this stage is indicated by the intersection of the curve $A B$ (indicated by solid line in figure1) and the vertical line representing percentage weight composition of Beryllium in the Beryllium-Copper phase diagram (Figure1). In this stage the dissolved beryllium precipitates as a beryllium rich (gamma) phase in the copper matrix along the grain boundaries. The formation of this precipitate leads to the increase in the strength of the specimen.

\section{Conventional Method Adopted}

The specimen is placed in a preheated muffle furnace for the required duration. It is then removed from the furnace and pulled from both ends using pliers, in order to straighten it before water quenching. The same steps are then repeated for Precipitation Hardening.

\section{Experimental Analysis}

An experiment was carried out on a Beryl-Co strip using the conventional method.

\subsection{Process Involved}

Step 1: The mill hardened material was heated to $650^{\circ} \mathrm{C}$ in a furnace for 17 minutes.

Step 2: It was then immediately quenched in a water bath and pulled using pliers in order to keep it in tension.

Step 3: Then it was heated in the furnace to $250^{\circ} \mathrm{C}$ for 25 minutes.

Step 4: Finally, it was water quenched again to room temperature.

\subsection{Observation}

Spring back effect of the specimen was reduced. Also, a thin layer of rust was formed due to oxidation. 


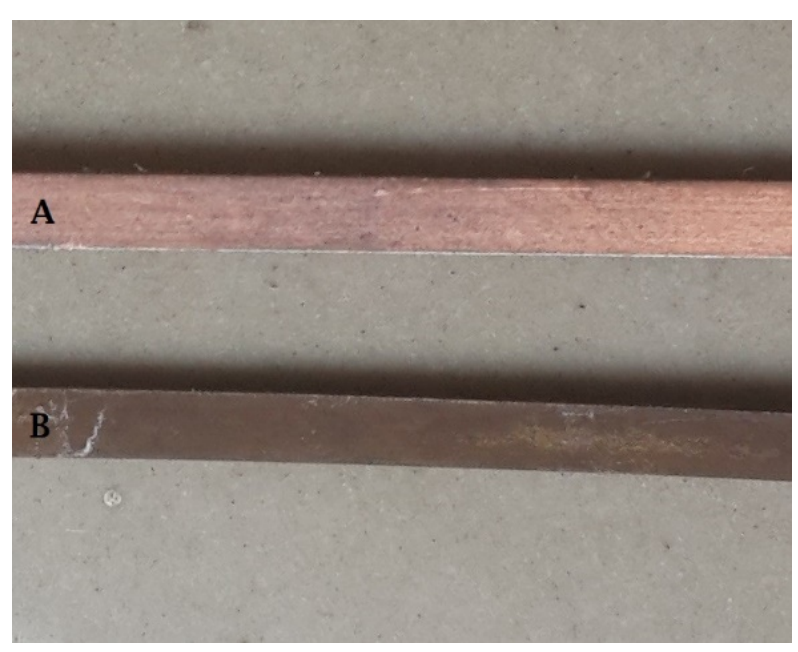

Figure 2. (A) Beryllium-Copper strip before heat treatment (B) Beryllium-Copper strip after heat treatment.

\section{Limitations of the Conventional Method}

During the course of heat treatment, the specimen has to be held in tension while quenching. However while carrying out heat treatment process by the conventional method there is rapid loss of heat from the specimen in the time that it takes to remove the it from the furnace and straighten it. During this time, the temperature falls below the critical limit (indicated by intersection of curve $\mathrm{AB}$ and the line representing percentage weight composition of Beryllium in figure1) before the specimen is quenched. Due to this there is a reduction in the yield strength of the material, making the process unsuccessful.

\section{Methods to Overcome the Limitations}

The following changes may be adopted to the conventional method in order to overcome the above mentioned limitations:

A. By developing an apparatus such that the specimen conducts heat to the surroundings at a much lower rate allowing it to be quenched before its temperature falls below the critical limit.

B. By using a heating method in which the specimen can be quenched directly after heating.

\subsection{Method 1: Flat Steel Rod Method.}

The antenna specimen is secured to a steel rod having a flat surface using kapton tapes. Kapton tapes are used because they remain stable over a wide range of temperatures. The contact between the steel rod and the BerylCo is avoided by putting a layer of kapton tape between them (as shown in figure3). The entire apparatus is then heated to the required temperature in a furnace. Since the two metals are in contact, heat will be conducted from the steel rod to the specimen through the kapton tapes. This lowers the rate of heat loss by the specimen. The entire apparatus is then water quenched while it is above the critical temperature and thus, its yield strength increases.

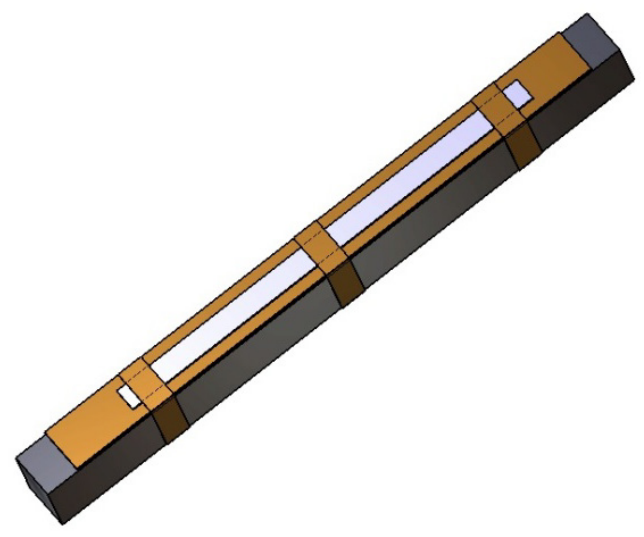

Figure 3. Apparatus showing the steel rod, Antenna and kapton tape

\subsection{Method 2: Circular Pipe Method}

Sometimes, commercially available muffle furnaces are not big enough to house the steel rod apparatus discussed above. In such cases, an alternate method can be used. The specimen is kept inside a hollow circular steel tube. It is attached to a projection at the top by using kapton tape (as shown in figure4), suspending it from the top. A suitable weight is attached to the bottom to keep the specimen under tension. The steel pipe is then heated externally to a temperature of around $800^{\circ} \mathrm{C}$ (well below the melting point of steel which is $1370^{\circ} \mathrm{C}$ ) for 15 minutes. The specimen is then quenched by pouring water from above and quickly released. It falls down vertically due to the attached weight. The same procedure is repeated for the precipitation hardening process.

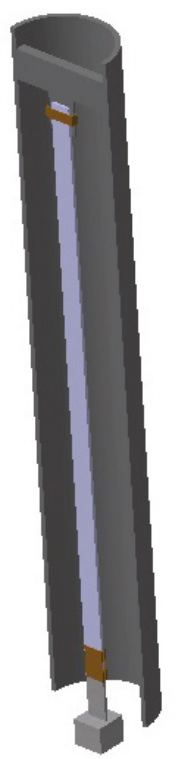

Figure 4. Cross Section of the steel tube showing the antenna specimen with the added weight. 


\section{Theoretical Evaluation}

A theoretical evaluation of the methods was carried out during which it was observed that in both the processes, the specimen is held in tension during the heat treatment process which is essential to enhance the spring back effect of the specimen. Furthermore both methods also allow for water quenching of the specimen before its temperature falls below the critical level.

\section{Advantages of the Proposed Methods}

The methods mentioned above address both the limitations of the conventional method, simultaneously i.e. rapid heat loss to the surroundings and keeping the specimen in tension during the process. The set-up required is very simple and can be constructed at a low cost. An added advantage is the flexibility of the apparatus as there is no restriction on the shape of the steel rod used in the first method or the diameter and material of the hollow tube used in the second. Additionally, the processes can be carried out over a wide range of temperatures. However, one disadvantage of circular pipe method is that since the process is carried out in the open, a thin layer of rust is formed over time. This problem is not serious as the rust layer can be scraped off without reducing the spring-back effect of the specimen.

\section{Conclusions}

Due to the rapid advancements in technology and miniaturization of satellites, Beryl-Co strips used for space applications are reducing in size. An experimental analysis carried out highlights the limitations of the conventional process when it comes to precipitation hardening of thin strips. These limitations can be overcome by adopting the new methods mentioned above as described in the theoretical evaluation.

\section{REFERENCES}

[1] CDA Publication No 54, "Beryllium Copper", 1962.

[2] AmitavaGuha, "PROPERTIES OF BERYLLIUM COPPER C17510 FOR HIGH CONDUCTIVITY APPLICATIONS", Brush Wellman Inc, 17876 St. Clair Ave, Cleveland, Ohio 4411 .

[3] Lee S. H, Saito Y, Sakai T \& Utsunomiya H, "Microstructures and mechanical properties of 6061 aluminum alloy processed by accumulative roll-bonding", Material Science Engineering A, 325 (2002), 228-35.

[4] Gra'zynaMr'owka-Nowotnik\& Jan Sieniawski, "Influence of heat treatment on the microstructure and mechanical properties of 6005 and 6082 aluminium alloys", Journal of Materials Processing Technology, 162 (2005), 367-372.

[5] K. Elleuch, S. Mezlini, N. Guermazi\& Ph. Kapsa, "Abrasive wear of aluminium alloys rubbed against sand", Journal of Wear, 261 (2006), 1316-1321.

[6] N. Kamp, N. Gao, M.J. Starink\& I. Sinclair, "Influence of grain structure and slip planarity on fatigue crack growth in low alloying artificially aged 2xxx aluminium alloys", International Journal of Fatigue, 29 (2007), 869-878.

[7] K.G. Basavakumar, P.G. Mukunda\& M. Chakraborty, "Impact toughness in $\mathrm{Al}-12 \mathrm{Si}$ and $\mathrm{Al}-12 \mathrm{Si}-3 \mathrm{Cu}$ cast alloys-Part 1: Effect of process variables and microstructure", International Journal of Impact Engineering, 35 (2008), 199-205.

[8] Wang D, Nia D. R \& Ma Z. Y, "Effect of pre-strain and two-step aging on microstructure and stress corrosion cracking of 7050 alloy", Material Science Engineering A, 143(2008), 298-303.

[9] Sangshik Kim, James T. Burns \& Richard P. Gangloff, "Fatigue crack formation and growth from localized corrosionin $\mathrm{Al}-\mathrm{Zn}-\mathrm{Mg}-\mathrm{Cu}$ " Engineering Fracture Mechanics, 76 (2009), 651-667.

[10] Mohammad Tajally, Zainul Huda \& H.H. Masjuki, "A comparative analysis of tensile and impact-toughness behavior of cold-worked and annealed 7075 aluminum alloy", International Journal of Impact Engineering, 37 (2010), 425-432. 